\title{
On CSO-Compact Space
}

\author{
Zina M. Kadem* \\ Jalal Hatem Hussein*
}

Date of acceptance 3/7/2008

\begin{abstract}
:
The aim of this paper is to introduces and study the concept of CSO-compact space via the notation of simply-open sets as well as to investigate their relationship to some well known classes of topological spaces and give some of his properties.
\end{abstract}

\section{Introduction and Preliminaries}

Let $(X, T)$ be a topological space. For a subset $S$ of $X$, the closure, the interior and the complement of $S$ with respect to $(X, T)$ will be denoted by $\boldsymbol{c l} S$, int $S$ and $\left(X-S=S^{c}\right)$ respectively. And let $\boldsymbol{w}$ be denoted the set of all positive integers. The subspace topology on a subset $S$ of a space $(X, T)$ is denoted by $T / S$.

Semi-open subsets were defined by Levine. Recall that $S$ is called semiopen [1] if $S \subset \operatorname{cl}($ int $S$ ). A semi-closed set is a set, whose complement is semiopen [2].

Recently there has been some interest in the notion of a simply-open subset of topological space $(X, T)$. According to Neubrunnovia [3] a subset $S$ of a space $(X, T)$ is called simply-open if it is $S=\mathrm{O} \cup \mathrm{N}$, where $\mathrm{O}$ is open and $\mathrm{N}$ is nowhere dense $(=\mathrm{nwd}$, int $(\mathrm{cl} \mathrm{N})=\Phi)$ subset of a space $(X, T)$. In [4] Ganster, Reilly and Vamanmurthy showed that a subset $S$ of a space $(X, T)$ is simply-open if and only if it is intersection of semi-open and semi-closed subsets of a space $(X, T)$. In [5] and [6] simply-open sets called as semi-locally closed set and NDB-set, respectively.

Nour [7] showed that a subset $S$ of a space $(X, T)$ is called regular open (resp. regular closed) if $S=$ int (cl $S$ ) (resp. $S=$ cl (int $S$ )). A set $S$ is called semi-regular [8] if it is both semi-open and semi-closed. Di Maio and Noiri [8] have pointed out that a subset $S$ of a space $(X, T)$ is called semi-regular if and only if there is regular open set $U$ such that $\mathrm{U} \subset S \subset \mathrm{cl} \mathrm{U}$. Cameron [9] used the term regular semi-open for a semi-regular set.

In 1994, Dlaska, Ergun and Ganster [10] introduced the class of countably S-closed space. A space $X$ is called countably $S$-closed if every countable cover of regular closed sets has finite subcover. In 1984, Porter and Woods [11] defined the concept of feebly compact spaces. A space $X$ is called feebly compact if every countable open cover of $X$ has finite subfamily such that the closures of whose members cover $X$.

Recall that $X$ is countably rscompact [12] ( resp. semi-countably compact [13], mildly compact [14] ) if every countable cover of $X$ by semiregular ( resp. semi-open, regular open) sets has a finite subcover.

The families of regular open(resp. regular closed) subsets of $(X, T)$ is denoted by $\boldsymbol{R O}(X, T)$ ( resp. $\boldsymbol{R C}(\boldsymbol{X}, \boldsymbol{T}))$. And $R O(X, T)$ is a base for a coarser topology $\boldsymbol{T}_{s}$ on $X$, called the semi-regularization topology on $X$.

Grossely and Hildebrand [15] defined a irresolute (resp. pre-semiopen) function as, a function $\mathrm{f}: X \rightarrow Y$, such that $X$ and $Y$ be a topological spaces, is said to be irresolute(resp. pre-semi-open) if and only if, for any semi-open subset $S$ of $Y$ we have, $\mathrm{f}^{-1}(S)$ is semi-open in $X$ (resp. for all $S \in$ $\boldsymbol{S O}(X), \quad \mathrm{f}(S) \in S O(Y))$ and they

\footnotetext{
* Department of Mathematics/ College of Science for Women/ University of Baghdad
} 
defined a semi - homeomorphism function between two topological spaces as, a function $\mathrm{f}: X \rightarrow Y$ is semihomeomorphism if and only if it is one-to-one, onto, irresolute, and presemi-open.

\section{The relation between CSO- compact space and some well-known classes of topological spaces}

We begin by defining the class of spaces we will study in this paper.

Definition 2.1. A topological space $(X, T)$ is called CSO-compact if every countable cover of $X$ by simplyopen subset of $X$ has a finite subcover.

Lemma2.2. Every semi-open subset of a space $(X, T)$ is simply-open set.

Proof. Let $A$ be a semi-open subset of a space $(X, T)$. By [1],there exist an open set $O$ such that, $\mathrm{O} \subset A \subset \mathrm{clO}$, then $A=\mathrm{O} \cup(\mathrm{A}-\mathrm{O})$. Let $\mathrm{B}=A-\mathrm{O}$, then $\mathrm{B} \subset(\mathrm{clO}-\mathrm{O})$. Now only we shall to do is to prove that $B$ is nwd in $X$, so we have, $\operatorname{int}(\mathrm{cl}(\mathrm{clO}-\mathrm{O}))=$ $\left[\operatorname{int}\left(\operatorname{cl}\left(\mathrm{clO} \cap \mathrm{O}^{\mathrm{c}}\right)\right] \subset\left[\operatorname{int}\left(\mathrm{clO} \cap \mathrm{clO}^{c}\right)\right]=\right.$ $\left[\operatorname{int}\left(\mathrm{clO} \cap(\operatorname{intO})^{\mathrm{c}}\right] \subset\left[\mathrm{clO} \cap \operatorname{int}(\mathrm{intO})^{\mathrm{c}}\right]\right.$ $=\left[\mathrm{clO} \cap(\mathrm{clO})^{\mathrm{c}}\right]=\Phi$. So clO-O is nwd but $\mathrm{B} \subset(\mathrm{clO}-\mathrm{O})$, therefore $\mathrm{B}$ is nwd. This shows the Lemman

By upper lemma we can prove the following result

Proposition 2.3. Every CSOcompact space is countably S-closed space .

Proof. Let $\left\{\mathbf{G}_{\mathbf{i}}: \quad \mathbf{i} \in \mathrm{I}\right\}$ be a countable cover of apace $X$ by regular closed sets and, but we know that every regular closed sets is semi open set so $\{\mathbf{G}: \mathbf{i} \in \mathrm{I}\}$ is countable cover of $X$ by semi-open set and so by simplyopen set by Lemma2.2 but $X$ is CSOcompact space so $\left\{\mathbf{G}_{\mathbf{i}}: \mathbf{i} \in \mathrm{I}\right\}$ have finite subcover
Lemma2.4. The closure of semi-open set is regularly closed set.

Proof. For let A be a semi-open set of a topological space $(X, T)$. Then $\mathrm{A} \subseteq \mathrm{cl}(\operatorname{int}(\mathrm{A}))$. Hence

$\operatorname{cl}(\mathrm{A}) \subseteq \mathrm{cl}(\mathrm{cl}(\operatorname{int} \mathrm{A}))=\mathrm{cl}(\operatorname{int}(\mathrm{A}))$ Which

implies $\quad \operatorname{cl}(\mathrm{A}) \subseteq \operatorname{cl}(\operatorname{int}(\mathrm{A})) \quad$ So $\operatorname{cl}(\mathrm{A})=\operatorname{cl}(\operatorname{int}(\mathrm{A})) . \quad$ But $\operatorname{cl}(\operatorname{int}(\overline{\mathrm{A}})) \subseteq$ $\overline{\mathrm{A}}$ and $\quad \operatorname{cl}(\operatorname{int}(\mathrm{A})) \subseteq \operatorname{cl}(\operatorname{int}(\operatorname{cl} \mathrm{A})) \quad$ so $\operatorname{cl} A=\operatorname{cl}(\operatorname{int}(\operatorname{cl} A))$ and therefore $\bar{A}$ is regular closed setm

Proposition 2.5. Every CSOcompact space is feebly compact.

Proof. Let $\left\{\mathbf{G}_{\mathbf{i}}: \quad \mathbf{i} \in \mathrm{I}\right\}$ be a countable cover of apace $X$ by open sets, but we know that every open set is semi-open and so $\left\{\mathbf{G}_{\mathbf{i}}: \mathbf{i} \in \mathrm{I}\right\}$ is countable semi-open cover of $X$, so by Lemma2.4 $\left\{\mathrm{clG}_{\mathbf{i}}: \mathbf{i} \in \mathrm{I}\right\}$ is regular closed cover of $X$ and so by Proposition $2.3\left\{\mathrm{clG}_{\mathbf{i}}: \mathbf{i} \in \mathrm{I}\right\}$ have finite subcover

In our next example we show that the converses of upper propositions need not be true.

Example 2.6. Let $X$ be the real line $R$ with a topology in which the only non-trivial open subsets are $\{0\},\{1\}$ and $\{0,1\}$ clearly $X$ has only a finite number of regular open and open sets, and hence $X$ is countably S-closed and feebly compact space. Note now that the countable cover $\{R-w$ \}$\cup\{\{1, \mathrm{n}\}: \mathrm{n} \in w\}$ by simply-open sets has no finite subcover, hence $X$ is not CSO-compact space.

The following proposition is very easy and the proof will not be given. 
Proposition 2.7. In a discrete space every feebly compact space is CSO-compact space.

Proposition 2.8. Every semiregular subset of a space $X$ is simplyopen.

Proof. Let A be a semi-regular subset of a space $X$ so $\mathrm{A}$ is open and so semi-open then by Lemma2.2 $\mathrm{A}$ is simply-open set.

Proposition 2.9. Every CSOcompact space is countably rscompact(resp. semi- countably compact, mildly compact).

Proof. The proof coming from the fact say that every cover of $X$ by semiregular ( resp. semi-open, regular open) is simply-open cover, but $X$ is CSO-compact space, so its have subcover

The cofinite topology will show that the converse of previous proposition need not be true.

under what condition the converse of above proposition showed be true? The following theorem will answer the question.

Theorem 2.10. Every semicountably compact space, in which every semi-closed set is semi-open, is CSO-compact space.

Proof. Let $A$ be a simply-open subset of a space $X$, then $\mathrm{A}=\mathrm{O} \cup \mathrm{N}$ where, $\mathrm{O}$ is open and $\mathrm{N}$ is nwd subsets of a space $X$, so $\mathrm{O}$ is open and $\mathrm{N}$ is semi-closed and so $\mathrm{N}$, by theorem's condition, is semi-open. But the union of two semi-open set is semi-open, so $A$ is semi-open. Now let $\bigcup_{i \in I} A_{i}$, such that $I$ is countable, is countably simplyopen cover of $X$ then $\bigcup_{i \in I} A_{i}$ is countably semi-open cover of $X$, but $X$ is semi- countably compact space then $\bigcup_{i \in I} A_{i}$ have a finite subcover of $X$

Lemma2.11. [10] Let $(X, T)$ be a space, then if $G \subset X$ is locally dense, i.e. $G \subset \operatorname{int}(\operatorname{cl} G)$, then $R C(G, T / G)=$ $\{F \cap A: F \in R C(X, T)\}$

Theorem 2.12. Let $(X, T)$ be a CSO-compact space, then if $G \in$ $R O(X, T)$, then $(G, T / G)$ countably Sclosed space.

Proof. Let $\quad\left\{A_{n:} n \in w\right\} \quad \subset$ $R C(G, T / G)$ be a cover of $G$. By lemma 2.10, for each $\mathrm{n} \in w, A_{\mathrm{n}}=G \cap F_{n}$ for some $F_{n} \in R C(X, T)$. Since $\left\{F_{n}: n \in w\right.$ \}$\cup\{X-G\}$ is a regular closed cover of $(X, T)$,but $(X, T)$ is a CSO-compact space and so countably $S$-closed space by Proposition 2.3, so there exists $m \in w$ such that $X=\{X-G\} \cup F_{1} \cup$ $F_{2} \cup \ldots \ldots \ldots . . . \cup \quad F_{m}$. Consequently, $G=$ $A_{1} \cup \ldots \ldots \cup A_{m}$ and thus $(G, T / G)$ countably $S$-closed space

\section{Characterization and properties of CSO-compact space}

Lemma3.1 [15] if f: $(X, T) \rightarrow$ $(Y, F)$ is homeomorphism, then $\mathrm{f}$ is semi-homeomorphism

Lemma3.2 [15] if f: $(X, T) \rightarrow$ $(Y, F)$ is semi-homeomorphism and $N \subset X$ is nwd in $X$, then $\mathrm{f}(N)$ is nwd in $Y$.

Now we are in a position to prove our main result.

Theorem 3.3 If f: $(X, T) \rightarrow(Y, F)$ is homeomorphism and $(Y, F)$ is CSOcompact space, then is $(X, T)$ CSOcompact. 
Proof. Let $\left\{G_{i=} O_{i} \cup N_{i}: i \in \mathrm{I}\right\}$ be a countable simply-open cover of $X$, such that $O_{i}$ is open and $N_{i}$ is nwd subsets of a space $X$. so by lemma3.1, that $\mathrm{f}$ is semi-homeomorphism, so by lemma3.2 we have $\mathrm{f}\left(N_{i}\right), i \in \mathrm{I}$, provide that I countable, is nwd subsets of a space $Y$, and since $\mathrm{f}$ is homeomorphism then $\mathrm{f}\left(O_{i}\right), i \in \mathrm{I}$ is open subsets of a space $Y$.

Therefore $\left\{\mathrm{f}\left(O_{i}\right) \cup \mathrm{f}\left(N_{i}\right), i \in \mathrm{I}\right\}$ is countable simply-open cover of a space $Y$. But $Y$ is CSO-compact space, hence there exists a finite subcover of $Y, \quad$ say $\quad\left\{\mathrm{f}\left(O_{\mathrm{i}_{1}}\right) \cup \mathrm{f}\left(N_{\mathrm{i}_{1}}\right), \quad \mathrm{f}\left(O_{\mathrm{i}_{2}}\right)\right.$ $\left.\cup \mathrm{f}\left(N_{\mathrm{i}_{2}}\right), \ldots \ldots, \mathrm{f}\left(O_{\mathrm{i}_{n}}\right) \cup \mathrm{f}\left(N_{\mathrm{i}_{n}}\right)\right\}$, such that $\bigcup_{j}^{n}\left\{\mathrm{f}\left(O_{\mathrm{i}_{j}}\right) \cup \mathrm{f}\left(N_{\mathrm{i}_{j}}\right)\right\}=Y$, hence $\left(\mathrm{f}\left(O_{\mathrm{i}_{1}}\right) \quad \cup \mathrm{f}\left(N_{\mathrm{i}_{1}}\right)\right) \cup \quad\left(\mathrm{f}\left(O_{\mathrm{i}_{2}}\right)\right.$ $\left.\cup \mathrm{f}\left(N_{\mathrm{i}_{2}}\right)\right) \cup \ldots \ldots \ldots \ldots \ldots . . . \quad \cup\left(\mathrm{f}\left(O_{\mathrm{i}_{n}}\right)\right.$ $\left.\cup \mathrm{f}\left(N_{\mathrm{i}_{n}}\right)\right)=Y$, therefore we have, $\mathrm{f}\left(O_{i_{1}} \cup N_{i_{1}} \cup \quad O_{i_{2}} \cup N_{i_{2}} \cup \ldots \ldots . . \cup\right.$ $\left.O_{i_{n}} \cup N_{i_{n}}\right)=Y$, but $G_{i=} O_{i} \cup N_{i}, \quad i \in \mathrm{I}$, then $\mathrm{f}\left(G i_{1} \cup G i_{2} \cup \ldots \ldots \ldots \cup G i_{n}\right)=$ $Y$, so $G i_{1} \cup G i_{2} \cup \ldots \ldots \ldots \cup G i_{n}=X$. And this shows the validity of this theorem

\section{References}

1. Levine N.L.,1963 .Semi-open sets and semi -continuity in topological space, Amer. math. Monthly , 70: 36-41.

2. Crossly S.G. and Hildebrand S.K., 1971 Semi-closure, Texas J. Sic., 22 : $99-112$

3. Neubrunnovia, 1975 On transfinite sequences of certain types of functions, Acta. Fac. Rer. Natur. Univ. Com. Math., 30: 121- 126.

4. Ganster M., Reilly I.L. and Vamanmurthy M.K., 1992 Remarks onLocally closed sets,
Mathematical Pannonica, 3(2): 107- 113.

5. Sundaram P. and Balachandran K.,1990 Semi generalized Locally closed sets in topological spaces, Preprint.

6. Dub K. K., G. L. chae and O. S. Pan war ,1984. Some properties of "S-connectedness between sets" and "Set s-connected mappings", Indian J. Pure Appl. Math., 15 (4): 343-354.

7. Nour T. M., 1995. Totally semi continuous functions, Indian $\mathrm{J}$. Pure Appl. Math., 26 (7): 675-678.

8. Di Maio and T. Noiri, 1987. On sclosed spaces, Indian J. Pure Appl. Math., 18,(3): 226-233

9. Cameron D.E., 1978. Properties of S-closed spaces, Proc. Amer. Math. Soc., 72, 581-586

10. Dlaska K. , N. Ergun and M.Ganster, 1994. Countably Sclosed spaces, Math. Slovaca, 44 (3): 337-34 8.

11. Porter J.R. and R.G. Woods, 1984. Feebly compact spaces, Martin's Axiom and "Diamond", Topology Proc., 9: 105-121.

12. Dontchev J. and M. Ganster, 1996. On covering spaces whith semiregular sets, Ricerche Mat., 45 (1): 229-245.

13. Dlaska K., N. Ergun and M.Ganster,1995. On the topology generated by semi-regular sets, Indian J. Pure Appl. Math., 25 (11), 1163-1170.

14. Singal M.K. and A. Mathur,.1979. A note on mildly compact spaces, Kyungpook Math. J. , , 165-168.

15. Crossly S.G. and S.K. Hildebrand,1972. Semi-topological property ,Fund Math., 74 : 233254. 
حول الفضاء المرصوص-CSO

$$
\text { زينة محمد كاظمث* }
$$

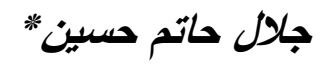

| قسم الرياضيات/ كلية العلوم للبنات/ جامعة بغداد

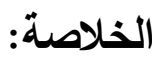

الهدف من هذا البحث هو تعريف و دراسة موضوع الفضاء المرصوص - CSO المعرف عن طريق

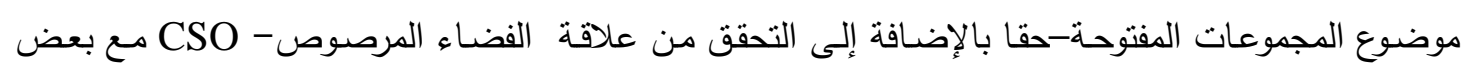
الفضاءات النوبولوجية المعروفة وذكر بعض خواصنه. 